\title{
Thorax: Annual Report October 1991-September 1992
}

It has been a busy year with a $16 \%$ increase in submitted manuscripts-a total of 621 being a record for the journal (see figure). Table 1 summarises the country of origin of manuscripts.

The time from first submission of the manuscript to a decision (reject or accept with revision) is shown in table 2 for both original papers and short reports. The overall mean time of 66 days for original papers represents the total time from receipt of the article, after which an associate editor is asked for the names of two reviewers; it includes the time taken for reviewer's comments and those of the associate editor to materialise and for the editor to review the manuscript and make his comments-that is, the interval between the day of arrival of the manuscript in the Thorax office to the day of postage back to the author. Clearly this figure includes only those manuscripts returned with a decision during the current year as only 446 of 621 have been dealt with so far. The turn round time for a revised manuscript has also been considerably reduced to a mean time of $22 \cdot 8$ days for original papers and 12 days for short reports (table 2).

Our rate of acceptance for original papers stands at $47.8 \%$ and $35 \%$ for short reports, which is similar to rates for the previous year.

Owing to the sustained acceptance rate and the increase in submitted manuscripts there is currently a mean delay of 5.6 months from acceptance to publication. The journal has, however, increased its pages to 96 per issue and we anticipate a reduction in this interval during the early part of 1993.

Thorax is publishing a list of reviewers for the first time this year. A considerable effort has been made to encourage reviewers to return manuscripts within 21 days. More than three quarters of the reviewers have responded to this and hence the overall verdict time for papers submitted to Thorax has come down sharply. We believe that the current verdict interval is acceptable and we wish to maintain this standard. The associate editors and the editor are extremely grateful to all of the reviewers approached during the last year and we trust that their efforts will continue so that we can maintain the high standard we are seeking for the journal.

Table 1 Country of original of submitted papers, 1991-2

\begin{tabular}{lc}
\hline Country & No of papers \\
\hline United Kingdom & 272 \\
Japan & 37 \\
Spain & 35 \\
Netherlands & 30 \\
Canada & 25 \\
USA & 23 \\
France & 17 \\
Irish Republic & 15 \\
Turkey & 15 \\
Israel & 14 \\
Australia & 14 \\
India & 10 \\
Germany & 9 \\
Italy & 8 \\
Switzerland & 8 \\
Sweden & 8 \\
Hong Kong & 8 \\
Austria & 7 \\
Belgium & 7 \\
China & 7 \\
Saudi Arabia & 6 \\
13 countries & $<5$ \\
\hline
\end{tabular}

Table 2 Decision times for submitted papers, 1991-2

\begin{tabular}{lcc}
\hline & $\begin{array}{l}\text { Original } \\
\text { papers }\end{array}$ & $\begin{array}{c}\text { Short } \\
\text { reports }\end{array}$ \\
\hline Number & 288 & 160 \\
Time to first decision & $66 \cdot 1$ & $54 \cdot 4$ \\
(mean(SD) days) & $(25 \cdot 6)$ & $(18 \cdot 4)$ \\
First quartile (days) & 50 & 43 \\
Papers resubmitted (n) & 65 & 21 \\
Time to final decision & $22 \cdot 8$ & $12 \cdot 2$ \\
(mean (SD) days) & $(22 \cdot 1)$ & $(6 \cdot 6)$ \\
\hline
\end{tabular}

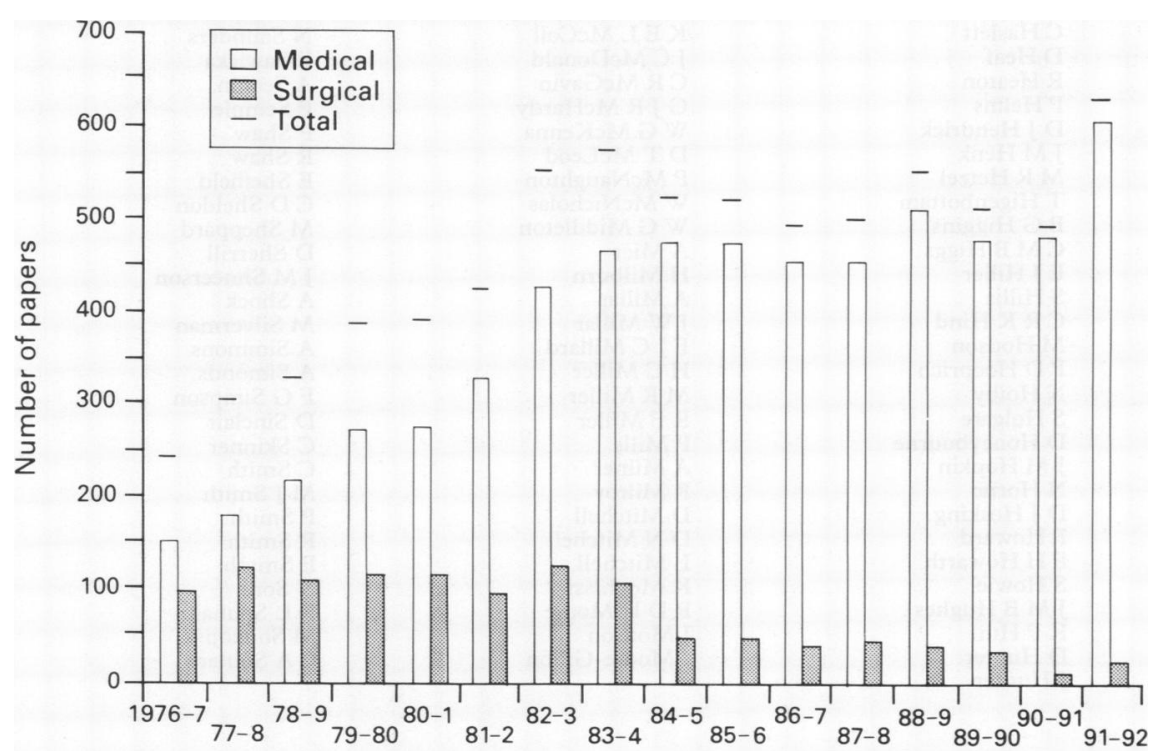

Annual total submission of manuscripts 
Reviewers used from 1 October 1991 to 30 September 1992

\begin{tabular}{|c|c|c|}
\hline 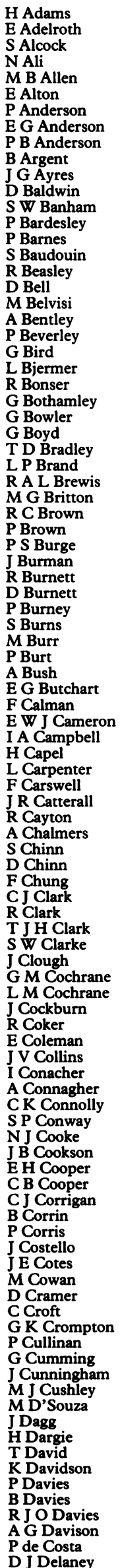 & 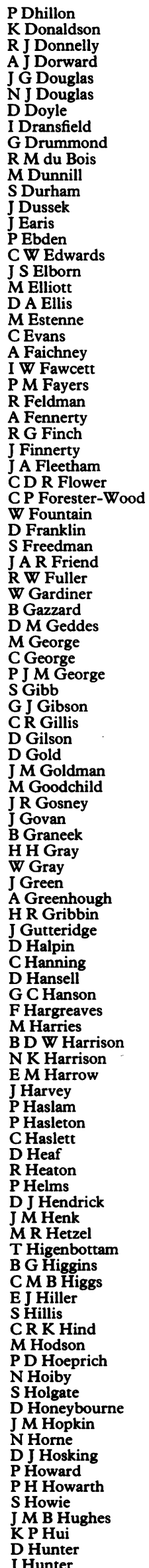 & 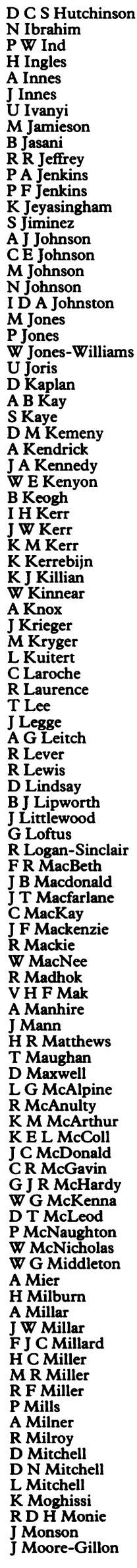 \\
\hline
\end{tabular}

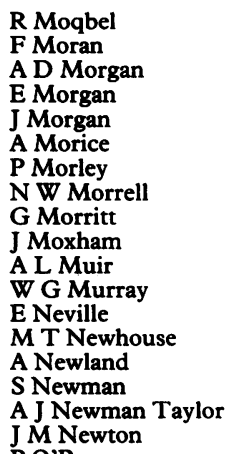

P O'Byrne

C O'Callaghan

B O'Connor

C O'Connor

G O'Connor

N Odom

L P Ormerod

G E Packe

C Page

R Page

C Pantin

J R Patel

D Pavia

I Pavord

A J Peacock

S J Pearce

M G Pearson

$S$ B Pearson

J Pepper

CPersson

R Phillips

L Poulter

J O Powell-Tuck

D Prakash

R J Prescott

N Pride

J G Prior

K Prowse

RO Quin

P J Rees

M Richardson

G Ridgway

B Riley

C M Roberts

G H Roberts

J A Roberts

N Roberts

A Robertson

M Roddie

M Roddie

M B Rubens

R Rudd

M Rudolp

G Russell

A Sampson

N Saunders

E Sawicka

A Seaton

P Semple

P Shaw

R Shaw

CD Sheldon

CD Sheldon

$M$ Sheppard

J M Shneerson

A Shock

M Silverman

A Simmons

A Simonds

F G Simpson

D Sinclair

C Skinne

C Smith

M J Smith

P Smith

E Smyth

N Soni

R L Souham

M Soukop
T Spector

D Stableforth

J Stark

M Stephens

P Sterk

G Sterling

M A Stern

S Stewart

R Stockley

R Stone

J Stradling

C Strange

T B Stretton

P Studdy

R D Sturrock

P P Sutton

A Swan

E M Sweet

R S Swindell

I Symington

R P Symonds

A R Tanser

A Tattersfield

B Taylor

W Taylor

N Thatcher

J St J Thomas

$M$ Thomas

S G Thompson

$N$ Thomson

$M J$ Tobin

J Tolmie

E Townsend

D Treacher

$T$ Treasure

D Trigger

D Turner

M A Turner

T Turner

C W G Turton
D O P Twentyma

D O P Twen

A S Vathenen

D Veale

E Waclawski

J Wales

E Walker

W Walker

W Wallace

E H Walters

$S$ Walters

$K$ P Wang

Th $\mathrm{H}$ Wankè

$M$ War

A Wardla

A Warley

J O Warner

$P$ Warren

J Waterlow

J Watson

B Watt

A K Webb

J R Webb

J A Wedzicha

D Weir

$S T$ Weiss

P H Weller

F C Wells

K Whaley

D Wheatley

W F Whimster

R White

J Wiggins

M Wilkinson

P Wilkinson

A Williams

A J William

A J Winning

$\mathrm{J} \mathbf{H}$ Winter

R Winter

A A Woodcock

M A Woodhead

N Wright

P H Wright

S Wynn

$\mathrm{N}$ van Zandwijk 